CERN-PPE/95-187

15th December 1995

\title{
POLARISATION OF VALENCE AND NON-STRANGE SEA QUARKS IN THE NUCLEON FROM SEMI-INCLUSIVE SPIN ASYMMETRIES
}

The Spin Muon Collaboration (SMC)

\begin{abstract}
We present a measurement of semi-inclusive spin asymmetries for positively and negatively charged hadrons from deep inelastic scattering of polarised muons on polarised protons and deuterons in the range $0.003<x<0.7$. From these asymmetries and the previously published inclusive spin asymmetries we determine, for the first time, the $x$-dependent spin distributions for $u p$ and down valence quarks and for non-strange sea quarks. We find that the first moments of the valence quark spin distributions are $\Delta u_{v}=1.01 \pm 0.19 \pm 0.14$ and $\Delta d_{v}=-0.57 \pm 0.22 \pm 0.11$. The spin distribution function of non-strange sea quarks is consistent with zero over the measured range of $x$ and the first moment is $\Delta \bar{u}=\Delta \bar{d}=-0.02 \pm 0.09 \pm 0.03$.
\end{abstract}


B. Adeva ${ }^{19}$, E. Arik ${ }^{2}$, A. Arvidson ${ }^{22}$, B. Badelek ${ }^{22,24}$, M.K. Ballintijn ${ }^{14}$, G. Bardin ${ }^{18}$, G. Baum ${ }^{1}$, P. Berglund ${ }^{7}$, L. Betev ${ }^{12}$, I.G. Bird ${ }^{18}$, R. Birsa ${ }^{21}$, P. Björkholm ${ }^{22}$, B.E. Bonner ${ }^{17}$, N. de Botton ${ }^{18}$, F. Bradamante ${ }^{21}$, A. Bressan ${ }^{21}$, S. Bültmann ${ }^{1}$, E. Burtin ${ }^{18}$, C. Cavata ${ }^{18}$, D. $\mathrm{Crabb}^{23}$, J. Cranshaw ${ }^{17}$, T. Çuhadar ${ }^{2}$, S. Dalla Torre ${ }^{21}$, R. van Dantzig ${ }^{14}$, A. Deshpande ${ }^{25}$, S. Dhawan ${ }^{25}$, C. Dulya ${ }^{3}$, A. Dyring ${ }^{22}$, S. Eichblatt ${ }^{17}$, J.C. Faivre ${ }^{18}$ D. Fasching ${ }^{16}$, F. Feinstein ${ }^{18}$, C. Fernandez ${ }^{8, g}$, B. Frois ${ }^{4,18}$, J.A. Garzon ${ }^{8, g}$, T. Gaussiran ${ }^{17}$, M. Giorgi ${ }^{21}$, E. von Goeler ${ }^{15}$, A. Gomez $^{19}$, G. Gracia ${ }^{19}$, N. de Groot ${ }^{14}$, M. Grosse Perdekamp ${ }^{3}$, E. Gülmez ${ }^{2}$, D. von Harrach ${ }^{10}$, T. Hasegawa ${ }^{13}$, P. Hautle ${ }^{4}$, N. Hayashi ${ }^{13}$, C.A. Heusch ${ }^{4, f}$, N. Horikawa ${ }^{13}$, V.W. Hughes ${ }^{25}$, G. Igo ${ }^{3}$, S. Ishimoto ${ }^{13}$, T. Iwata ${ }^{13}$, E.M. Kabuß ${ }^{10}$, T. Kageya ${ }^{13}$, A. Karev ${ }^{9}$, H.J. Kessler ${ }^{5}$, T.J. Ketel ${ }^{14}$, A. Kishi ${ }^{13}$, Yu. Kisselev ${ }^{9}$, L. Klostermann ${ }^{14}$, D. Krämer ${ }^{1}$, V. Krivokhijine ${ }^{9}$, W. Kröger ${ }^{4, f}$,

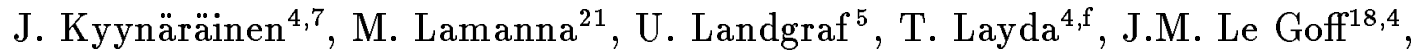
F. Lehar ${ }^{18}$, A. de Lesquen ${ }^{18}$, J. Lichtenstadt ${ }^{20}$, M. Litmaath ${ }^{14}$, S. Lopez-Ponte ${ }^{8, g}$, M. Lowe ${ }^{17}$, A. Magnon ${ }^{18}$, G.K. Mallot ${ }^{10}$, F. Marie ${ }^{18}$, A. Martin ${ }^{21}$, J. Martino ${ }^{18}$, T. Matsuda ${ }^{13}$, B. Mayes ${ }^{8}$, J.S. McCarthy ${ }^{23}$, K. Medved ${ }^{9}$, G. van Middelkoop ${ }^{14}$, D. Miller ${ }^{16}$, K. Mori ${ }^{13}$, J. Moromisato ${ }^{15}$, A. Nagaitsev ${ }^{9}$, J. Nassalski ${ }^{24,4}$, L. Naumann ${ }^{4, z}$, T.O. Niinikoski ${ }^{4}$, J.E.J. Oberski ${ }^{14}$, A. Ogawa ${ }^{13}$, C. Ozben ${ }^{2}$ A. Penzo ${ }^{21}$, C. Perez ${ }^{19}$,

F. Perrot-Kunne ${ }^{18}$, D. Peshekhonov ${ }^{9}$, R. Piegaia ${ }^{4}$, L. Pinsky ${ }^{8}$, S. Platchkov ${ }^{18}$, M. Plo ${ }^{19}$, D. Pose ${ }^{9}$, H. Postma ${ }^{14}$, J. Pretz ${ }^{10}$, T. Pussieux ${ }^{18}$, J. Pyrlik ${ }^{8}$, I. Reyhancan ${ }^{2}$, A. Rijllart ${ }^{4}$, J.B. Roberts ${ }^{17}$, S. Rock ${ }^{4}$, M. Rodriguez ${ }^{22}$, E. Rondio ${ }^{24}$, A. Rosado ${ }^{12}$, I. Sabo ${ }^{20}$, J. Saborido ${ }^{19}$, A. Sandacz ${ }^{24}$, I. Savin ${ }^{9}$, P. Schiavon ${ }^{21}$, K.P. Schüler ${ }^{25}$, R. Segel ${ }^{16}$, R. Seitz ${ }^{10}$, Y. Semertzidis ${ }^{4}$, S. Sergeev ${ }^{9}$ F. Sever ${ }^{14,18}$, P. Shanahan ${ }^{16}$, E. P. Sichtermann ${ }^{14}$, G. I. Smirnov ${ }^{9}$, A. Staude ${ }^{12}$, A. Steinmetz ${ }^{10}$, U. Stiegler ${ }^{4}$, H. Stuhrmann ${ }^{6}$, M. Szleper ${ }^{24}$, K.M. Teichert ${ }^{12}$, F. Tessarotto ${ }^{21}$, M. Velasco ${ }^{16}$, J. Vogt $^{12}$, R. Voss ${ }^{4}$, R. Weinstein ${ }^{8}$, C. Whitten ${ }^{3}$, R. Windmolders ${ }^{11}$, R. Willumeit ${ }^{6}$, W. Wislicki ${ }^{24}$, A. Witzmann ${ }^{5}$, A.M. Zanetti ${ }^{21}$, J. Zhao ${ }^{6}$ 
Recent results from the polarised inclusive deep inelastic scattering of leptons by protons and deuterons $[1,2,3,4,5]$ show that the quark spins contribute only about $20 \%$ to the nucleon spin with a relatively large negative contribution of about $-10 \%$ from the strange quarks. These conclusions are based on the analysis of the first moments of the spin-dependent structure functions $g_{1}(x)$ of the proton and deuteron in the framework of the quark parton model (QPM). More detailed information on the spin structure of the nucleon can be obtained from polarised semi-inclusive deep inelastic scattering, where in addition to the scattered lepton also hadrons are detected. An early theoretical pre-

1) University of Bielefeld, Physics Department, 33501 Bielefeld, Germany ${ }^{n}$

2) Bogaziçi University and Çekmece Nuclear Research Center, Istanbul Technical University, Istanbul University, Istanbul, Turkey ${ }^{\circ}$

3) University of California, Department of Physics, Los Angeles, $90024 \mathrm{CA}, \mathrm{USA}^{\mathrm{p}}$

4) CERN, 1211 Geneva 23, Switzerland

5) University of Freiburg, Physics Department, 79104 Freiburg, Germany ${ }^{\text {n}}$

6) GKSS, 21494 Geesthacht, Germany ${ }^{\text {n }}$

7) Helsinki University of Technology, Low Temperature Laboratory and Institute of Particle Physics Technology, Otakaari 3A, 02150 Finland

8) University of Houston, Department of Physics, Houston, 77204-5504 TX, and Institute for Beam Particle Dynamics, Houston, 77204-5506 TX, USA ${ }^{\mathrm{p}, \mathrm{q}}$

9) JINR, Laboratory of Particle Physics, Dubna, Russia

10) University of Mainz, Institute for Nuclear Physics, 55099 Mainz, Germany ${ }^{\text {n }}$

11) University of Mons, Faculty of Science, 7000 Mons, Belgium

12) University of Munich, Physics Department, 80799 Munich, Germany ${ }^{\mathrm{n}}$

13) Nagoya University, Department of Physics, Furo-Cho, Chikusa-Ku, 464 Nagoya, Japan ${ }^{\mathrm{r}}$

14) NIKHEF, Delft University of Technology, FOM and Free University, 1009 AJ Amsterdam, The Netherlands ${ }^{s}$

15) Northeastern University, Department of Physics, Boston, 02115 MA, USA ${ }^{q}$

16) Northwestern University, Department of Physics, Evanston, 60208 IL, USA ${ }^{\mathrm{p}, \mathrm{q}}$

17) Rice University, Bonner Laboratory, Houston, 77251-1892 TX, USA ${ }^{\mathrm{p}}$

18) DAPNIA, C.E. Saclay, 91191 Gif-sur-Yvette, France ${ }^{t}$

19) University of Santiago, Department of Particle Physics, 15706 Santiago de Compostela, Spain ${ }^{\mathrm{u}, \mathrm{y}}$

20) Tel Aviv University, School of Physics, 69978 Tel Aviv, Israel ${ }^{\mathrm{v}}$

21) INFN Trieste and University of Trieste, Department of Physics, 34127 Trieste, Italy

22) Uppsala University, Department of Radiation Sciences, 75121 Uppsala, Sweden

23) University of Virginia, Department of Physics, Charlottesville, 22901 VA, USA ${ }^{\mathrm{p}}$

24) Soltan Institute for Nuclear Studies and Warsaw University, 00681 Warsaw, Poland ${ }^{\text {w }}$

25) Yale University, Department of Physics, New Haven, 06511 CT, USA ${ }^{p}$

f) Permanent address: University of California, Institute of Particle Physics, Santa Cruz, 95064 CA, USA

g) Permanent address: University of Santiago, Department of Particle Physics, 15706 Santiago de Compostella, Spain

n) Supported by the Bundesministerium für Bildung, Wissenschaft, Forschung und Technologie

o) Partially supported by TUBITAK and the Centre for Turkish-Balkan Physics Research and Application (Bogaziçi University)

p) Supported by the U.S. Department of Energy

q) Supported by the U.S. National Science Foundation

r) Supported by Ishida Foundation, Mitsubishi Foundation and Monbusho International Science Research Program

s) Supported by the National Science Foundation (NWO) of the Netherlands

t) Supported by the Commissariat à l'Energie Atomique

u) Supported by Comision Interministerial de Ciencia y Tecnologia

v) Supported by the Israel Science Foundation.

w) Supported by the KBN SPUB/P3/209/94

y) Supported by Xunta de Galicia 
diction for hadron asymmetries in polarised deep inelastic lepton-hadron scattering was made in ref. [6]. The first measurement of these asymmetries was done by EMC with a polarised proton target [7]. We report on our measurement of semi-inclusive asymmetries of spin-dependent cross sections for muoproduction of positive and negative hadrons from polarised protons and deuterons. These semi-inclusive asymmetries and our already published inclusive asymmetries $[1,2,3]$ are analysed together within the QPM and allow us to separate for the first time the spin distributions of the valence $u p$ and down quarks and of the non-strange sea quarks and to determine their dependence on the Bjorken scaling variable $x$.

Our experimental set up at the CERN muon beam consists of three major components: a polarised target, a spectrometer and a muon beam polarimeter. Positive muons of 100 and $190 \mathrm{GeV}$ were used. The muon beam polarisation was determined from the shape of the energy spectrum of positrons from the muon decays. It was found to be $-0.82 \pm 0.06$ [1] and $-0.81 \pm 0.04$ [2] for the 100 and $190 \mathrm{GeV}$ beams, respectively. The target consists of two cells filled with butanol or deuterated butanol. The two cells are polarised in opposite directions by dynamic nuclear polarisation. The average polarisations were $0.86 \pm 0.03$ for protons [2] and $0.47 \pm 0.03$ for deuterons $[3,8]$. Events originating from both cells were recorded simultaneously and the spin orientations were reversed every five hours. The scattered muon and produced charged particles were measured in the SMC spectrometer [2]. Muons were identified behind a $2 \mathrm{~m}$ thick iron absorber wall. The spectrometer has good acceptance for hadrons with $z \equiv E_{\text {had }} / \nu$ above 0.1 .

The determination of semi-inclusive asymmetries requires the separation of hadrons from electrons which originate mainly from photon conversions. For this purpose we used a calorimeter [9]. Its electromagnetic part amounts to 20 radiation lengths which is sufficient to contain entirely high energy electromagnetic showers. The total thickness of the calorimeter is 5.5 nuclear interaction lengths. For each shower the ratio $r_{e m}$ of the energy deposited in the electromagnetic part to the total deposited energy is calculated. Electrons are eliminated by requiring $r_{e m}<0.8$.

A cut $Q^{2}>1 \mathrm{GeV}^{2}$ is applied. The range of $x$ covered is $0.003<x<0.7$ and the average $Q^{2}$ is $10 \mathrm{GeV}^{2}$. For a hadron a cut $z>0.2$ is found to be an optimum considering the effective tagging of the struck quark at large $z$ and significant statistics from small $z$.

After applying kinematic and geometrical cuts we have about $4.5 \times 10^{6}$ deep inelastic events on the deuteron at $100 \mathrm{GeV}, 4.5 \times 10^{6}$ events on the proton at $190 \mathrm{GeV}$ and $6 \times 10^{6}$ events on the deuteron at $190 \mathrm{GeV}$. After cuts on $z$ and $r_{e m}$ the corresponding hadron samples amount to $1.4 \times 10^{6}, 1.2 \times 10^{6}$ and $1.6 \times 10^{6}$ charged hadrons, mostly pions, where the ratio of positive to negative hadrons is about 1.3 .

The inclusive cross section $\sigma^{\mu}$ and the semi-inclusive cross section for the production of a positive (negative) hadron, $\sigma^{+(-)}$, are related to the number of deep inelastic events $N^{\mu}$ and the number of hadrons $N^{+(-)}$. In the QPM the semi-inclusive cross sections factorise in terms of the quark distribution functions $q\left(x, Q^{2}\right)$ and quark fragmentation functions $D_{q}^{h}\left(z, Q^{2}\right)$

$$
\frac{1}{\sigma^{\mu}} \frac{d \sigma^{+(-)}}{d z}=\frac{1}{N^{\mu}} \frac{d N^{+(-)}}{d z}=\frac{\sum_{q, h} e_{q}^{2} q\left(x, Q^{2}\right) D_{q}^{h}\left(z, Q^{2}\right)}{\sum_{q} e_{q}^{2} q\left(x, Q^{2}\right)},
$$

where the hadron $h$ can be $\pi^{+}, K^{+}$or $p$ for $\sigma^{+}$and $\pi^{-}, K^{-}$or $\bar{p}$ for $\sigma^{-}$. The fragmentation function $D_{q}^{h}$ represents the probability that a struck quark with a flavour $q$ fragments into

z) Deceased 
a hadron $h$.

The semi-inclusive asymmetries of the spin-dependent virtual photon absorption cross sections for production of positive (negative) hadrons on protons and deuterons are defined as

$$
A_{1 p}^{+(-)}=\frac{\sigma_{1 / 2}^{+(-)}-\sigma_{3 / 2}^{+(-)}}{\sigma_{1 / 2}^{+(-)}+\sigma_{3 / 2}^{+(-)}}, \quad A_{1 d}^{+(-)}=\frac{\sigma_{0}^{+(-)}-\sigma_{2}^{+(-)}}{\sigma_{0}^{+(-)}+\sigma_{2}^{+(-)}} .
$$

The indices $1 / 2,3 / 2,0$ and 2 refer to the total spin projection in the direction of the virtual photon momentum.

Yields of positive and negative hadrons for each target cell and each spin configuration are given by

$$
N^{+(-)}=n \Phi \sigma^{+(-)} a^{+(-)}\left[1-f^{+(-)} P_{B} P_{T} D A_{1}^{+(-)}\right],
$$

where $n$ is the area density of nucleons in the target, $\Phi$ the beam flux, $\sigma^{+(-)}$is the unpolarised cross section for muoproduction of charged hadrons and $a^{+(-)}$the acceptance of the spectrometer for the simultaneous detection of the scattered muon and the charged hadrons. The symbols $P_{B}$ and $P_{T}$ denote the beam and the target polarisations. The depolarisation factor $D$ depends on the event kinematics and on the ratio $R$ of longitudinal to transverse unpolarised virtual photon cross sections [10]. The dilution factors $f^{+(-)}$are calculated separately for the proton and the deuteron target. The yields $N^{+(-)}$are measured before (B) and after (A) polarisation reversal for the upstream (U) and the downstream (D) target. The asymmetries $A_{1}^{+(-)}$are obtained from the ratio $\left(N_{U}^{B} N_{D}^{A}\right) /\left(N_{D}^{B} N_{U}^{A}\right)$ where the quantities $n, \Phi$ and $\sigma^{+(-)}$cancel out. The acceptances also cancel if their ratio for the two target cells is the same before and after polarisation reversal $\left(a_{U}^{B} / a_{D}^{B}=a_{U}^{A} / a_{D}^{A}\right)$. Radiative corrections to the asymmetries are small and therefore neglected.

The semi-inclusive asymmetries $A_{1}^{+(-)}$for positive and negative hadrons from deuteron and proton targets are given in fig. 1 and tab. 1 . The asymmetries $A_{1 p}^{+(-)}$are in agreement with those measured by the EMC [7]. In addition to the uncertainties discussed in refs. $[2,3]$ there is also a contribution to the systematic error from the variation in time of the spectrometer acceptance for hadrons. The effect on the asymmetries from secondary interactions of hadrons was found to be negligible.

In the QPM, neglecting possible gluon contributions, the inclusive virtual photon absorption cross sections can be written in terms of the quark spin distribution functions $\Delta q=q^{\uparrow}-q^{\downarrow}$, where $q^{\dagger(\downarrow)}\left(x, Q^{2}\right)$ is the distribution of quarks with flavour $q$ with spin parallel (antiparallel) to the nucleon spin. For inclusive cross sections the virtual photon absorption asymmetry is

$$
A_{1}\left(x, Q^{2}\right)=\frac{\sum_{q} e_{q}^{2} \Delta q\left(x, Q^{2}\right)}{\sum_{q} e_{q}^{2} q\left(x, Q^{2}\right)},
$$

where $e_{q}$ is the fractional charge of the quark and $q=u, d, s, \bar{u}, \bar{d}, \bar{s}$. With the assumption that fragmentation functions do not depend on the quark helicity [11], the virtual photon spin asymmetries for the production of positive and negative hadrons can be written

$$
A_{1}^{+(-)}\left(x, Q^{2}\right)=\frac{\sum_{q, h} e_{q}^{2} \Delta q\left(x, Q^{2}\right) D_{q}^{h}\left(Q^{2}\right)}{\sum_{q, h} e_{q}^{2} q\left(x, Q^{2}\right) D_{q}^{h}\left(Q^{2}\right)}
$$

where $D_{q}^{h}\left(Q^{2}\right)=\int_{0.2}^{1} d z D_{q}^{h}\left(z, Q^{2}\right)$. Following the usual convention all quark distributions refer to the proton. By using isospin symmetry the up and down quarks and antiquarks 
in the neutron are replaced by down and up quarks and antiquarks in the proton. The deuteron cross section is considered to be the sum of the proton and the neutron cross sections, corrected for the D-state of the deuteron as in ref. [3].

The MRS parametrisation [12] is used for unpolarised quark distributions. There are 36 fragmentation functions corresponding to three quarks, three antiquarks and six charged hadrons. The fragmentation functions of non-strange quarks into pions can be obtained from the EMC measurements [13] by using charge conjugation and isospin symmetry. Only these fragmentation functions give substantial contributions to the asymmetries. A further reduction of the number of independent fragmentation functions requires assumptions for unfavoured (e.g. $D_{d}^{\left[K^{-}=\bar{u} s\right]}=D_{\bar{d}}^{\left[K^{-}=\bar{u} s\right]}$ ) and favoured (e.g. $D_{s}^{\left[K^{-}=\bar{u} s\right]}=$ $D_{d}^{\left[\pi^{-}=\bar{u} d\right]}$ ) fragmentation. Finally the number of independent fragmentation functions is reduced from 36 to 6 .

The asymmetries are assumed to be independent of $Q^{2}$, consistent with our observation for the inclusive case [14]. Quark distribution functions and fragmentation functions are evaluated at $Q^{2}=10 \mathrm{GeV}^{2}$.

For each bin of $x$ eqs. (4) and (5) for proton and deuteron targets constitute a system of $2+4=6$ linear equations for $2 \times 3=6$ spin distributions of quarks and antiquarks. Since $D_{q}^{h} \neq D_{\bar{q}}^{h}, \Delta q(x)$ and $\Delta \bar{q}(x)$ have different weights and can be separated. For each flavour the valence and sea spin distributions are defined as $\Delta q_{v}(x)=\Delta q(x)-\Delta \bar{q}(x)$ and $\Delta q_{s e a}(x)=2 \Delta \bar{q}(x)$. The weight of the strange quark spin distribution functions in these equations is small and therefore they cannot be determined. We assume $\Delta s(x)=\Delta \bar{s}(x) \propto$ $\bar{s}(x)$ with $\int_{0.003}^{0.7} d x(\Delta \bar{s}(x)+\Delta s(x))=-0.12$, according to [3]. We also assume $\Delta \bar{u}(x)=$ $\Delta \bar{d}(x)$ and denote it by $\Delta \bar{q}(x)$. Measurements of the Gottfried sum rule [15] indicating that $\bar{u}(x) \neq \bar{d}(x)$ can be qualitatively explained by the pionic contribution to the quark sea [16]. The same reasoning applied for the polarised case gives $\Delta \bar{u}(x)=\Delta \bar{d}(x)=0[17]$. As mentioned above, in the present analysis we will only assume $\Delta \bar{u}(x)=\Delta \bar{d}(x)$. The effect of this assumption will be discussed below. The three unknown spin distributions $\Delta u_{v}(x)$, $\Delta d_{v}(x)$ and $\Delta \bar{q}(x)$ are evaluated from the system of six equations by the least squares method using the full covariance matrix between asymmetries. The values of correlation coefficients between semi-inclusive and inclusive asymmetries are given in tab. 2 . The average $\chi^{2} / d$.o.f. for the 12 bins of $x$ is $4.0 / 3$.

The distributions $x \Delta q(x)$ evaluated at $Q^{2}=10 \mathrm{GeV}^{2}$ are given in tab. 1 and in fig. 2 (closed circles). We observe that $\Delta u_{v}(x)$ is positive while $\Delta d_{v}(x)$ is negative. The spin distribution of the non-strange sea $\Delta \bar{q}(x)$ is compatible with zero over the full range of $x$.

The uncertainty of these results is dominated by the statistical error. The main contributions to the systematic errors are due to the target and beam polarisations, the variation of acceptances with time, the statistical errors on the fragmentation functions and the uncertainty on the unpolarised quark distributions. The latter is estimated from the difference between the MRS [12] and GRV [18] parametrisations. We varied the assumptions about the favoured and unfavoured fragmentation functions discussed above by a factor 0.5 to 2 and found the effect on the quark spin distributions to be smaller than $10 \%$ of the overall systematic error. Errors coming from the assumption on $\Delta s(x)$ are small. Also negligible errors arise from the difference in acceptance for pions, kaons and protons due to different absorption in the target and different angular distributions. A fit with $\Delta \bar{u}(x)$ and $\Delta \bar{d}(x)$ determined independently substantially increases the statistical uncertainties and we find $\Delta \bar{u}(x)=\Delta \bar{d}(x)$ within statistical errors. 
The statistical error on $\Delta \bar{q}(x)$ in the region $x>0.2$ is larger than the constraint from the unpolarized distribution, $|\Delta \bar{q}(x)| \leq \bar{q}(x)$ (cf. fig. 2c). In this region, in order to reduce the statistical errors on $\Delta u_{v}(x)$ and $\Delta d_{v}(x)$, we set $\Delta \bar{q}(x)=0$ before solving the system of equations. The results are shown as open circles in fig. 2 . We computed also the results using $\Delta \bar{q}(x)= \pm \bar{q}(x)$ and included the difference in the systematic errors.

In the QPM and with the assumption $\Delta \bar{u}(x)=\Delta \bar{d}(x)$, the quantities $6\left[g_{1}^{p}(x)-g_{1}^{n}(x)\right]$ and $\Delta u_{v}(x)-\Delta d_{v}(x)$ are equal. The former is obtained from inclusive data only while the latter can be extracted from semi-inclusive asymmetries of eqs. (5) alone. The data are in good agreement, as seen in fig. 3, showing the internal consistency of the present analysis.

An alternative approach to the analysis of the semi-inclusive asymmetries was developed in ref. [11]. The asymmetries are determined from the differences of cross sections for the positively charged and the negatively charged hadrons as

$$
A_{1 p}^{+-}=\frac{\left(\sigma_{1 / 2}^{+}-\sigma_{1 / 2}^{-}\right)-\left(\sigma_{3 / 2}^{+}-\sigma_{3 / 2}^{-}\right)}{\left(\sigma_{1 / 2}^{+}-\sigma_{1 / 2}^{-}\right)+\left(\sigma_{3 / 2}^{+}-\sigma_{3 / 2}^{-}\right)}, \quad A_{1 d}^{+-}=\frac{\left(\sigma_{0}^{+}-\sigma_{0}^{-}\right)-\left(\sigma_{2}^{+}-\sigma_{2}^{-}\right)}{\left(\sigma_{0}^{+}-\sigma_{0}^{-}\right)+\left(\sigma_{2}^{+}-\sigma_{2}^{-}\right)} .
$$

They are functions of the valence quark distributions only

$$
A_{1 p}^{+-}=\frac{4 \Delta u_{v}-\eta \Delta d_{v}}{4 u_{v}-\eta d_{v}}, \quad A_{1 d}^{+-}=\frac{\Delta u_{v}+\Delta d_{v}}{u_{v}+d_{v}}
$$

with $\eta$ calculated from fragmentation functions. The asymmetry $A_{1 d}^{+-}$does not depend on fragmentation functions whereas $A_{1 p}^{+-}$is only weakly sensitive to assumptions about fragmentation functions. Since the hadrons are not identified in the present experiment the factor $\eta$ has to be evaluated by averaging relevant fragmentation functions and is found to be about 0.5 for $z>0.2$. However, the spectrometer acceptance is different for positive and negative hadrons, and the ratio of these acceptances does not cancel out in the asymmetries (6) expressed in terms of the hadron yields. We measured this ratio in dedicated runs with a beam of oppositely charged muons and with a reversed field direction in the spectrometer magnet. It deviates from unity only for $x<0.02$ and by less then $15 \%$. The quark spin distribution functions $\Delta u_{v}(x)$ and $\Delta d_{v}(x)$ obtained using the asymmetries $A_{1}^{+-}$(eqs. (7)) are shown in fig. 4 with open circles. They are in good agreement with those determined from $A_{1}^{+}$and $A_{1}^{-}$(eq. (5)) (closed circles) without the constraint $\Delta \bar{u}(x)=\Delta \bar{d}(x)$. The results from the two methods are strongly correlated. Our conclusions concerning the quark spin distributions and their integrals are based on the analysis of the asymmetries $A_{1}^{+}$and $A_{1}^{-}$(eqs. (2)).

In order to calculate first moments $\Delta q$ of the quark spin distributions, $\Delta q=$ $\int_{0}^{1} d x \Delta q(x)$, we need extrapolations of $\Delta q(x)$ to unmeasured regions of $x$. We assume that the polarisation, $\Delta q_{v}(x) / q_{v}(x)$, in the unmeasured region is equal to its average value in the measured region. A $100 \%$ systematic error is assigned to these extrapolations. Contributions from the unmeasured large- $x$ region are found to be negligible. Such a method cannot be used for the small- $x$ extrapolation of $\Delta \bar{q}(x)$ since the integral of the unpolarized sea quark distribution diverges at small $x$. Since we do not observe any $x$-dependence for $\Delta \bar{q}(x)$, we fit it to a constant which we extrapolate to $x=0$. We obtained a negligible contribution to the integral. We estimate the systematic uncertainty from the error of the fit to the first two small- $x$ points only. The resulting integrals over the measured and unmeasured regions are given in tab. 3 together with the statistical and systematic errors. The integrals of quark spin distributions are $\Delta u_{v}=1.01 \pm 0.19 \pm 0.14$, 
$\Delta d_{v}=-0.57 \pm 0.22 \pm 0.11$ and $\Delta \bar{q}=-0.02 \pm 0.09 \pm 0.03$. The release of the constraint $\Delta \bar{u}(x)=\Delta \bar{d}(x)$ modifies mostly $\Delta d_{v}$ which becomes close to zero with a statistical error of 0.46 , while $\Delta u_{v}$ is little affected.

To summarize, we measured semi-inclusive asymmetries and determined, for the first time, the spin distribution functions $\Delta u_{v}(x), \Delta d_{v}(x)$ and $\Delta \bar{q}(x)$ of the valence and the non-strange sea quarks. Within the statistical accuracy the polarisations $\Delta q(x) / q(x)$ seem to be independent of $x$. The results indicate polarisations $\Delta u_{v} / u_{v}=0.5 \pm 0.1$ and $\Delta d_{v} / d_{v}=-0.6 \pm 0.2$. The values of $\Delta u_{v}(x)-\Delta d_{v}(x)$ derived from the semi-inclusive asymmetries alone agree well with the QPM interpretation of $g_{1}^{p}(x)-g_{1}^{n}(x)$ determined from the inclusive asymmetries. The total spin contribution from all non-strange sea quarks is $4 \Delta \bar{q}=-\mathbf{0 . 0 8} \pm \mathbf{0 . 3 6}$. The spin distribution function of the sea quarks $\Delta \bar{q}(x)$ is compatible with zero over the full measured range of $x$. The total spin carried by nonstrange quarks $\Delta \Sigma-2 \Delta \bar{s}=\Delta u_{v}+\Delta d_{v}+4 \Delta \bar{q}$ is consistent with the value deduced from first moments of $g_{1}(x)[3,5]$.

Our results show a sizeable polarisation of valence quarks, in particular at small $x$, which is consistent with the observation of $g_{1}^{n} \neq g_{1}^{p}$ at the smallest $x$ in the SMC data [3]. In the same region we observe that the spin contribution of the non-strange sea is small and consistent with zero, although the unpolarised sea is large. 
[1] SMC, B.Adeva et al., Phys.Lett. B302(1993)533

[2] SMC, D.Adams et al., Phys.Lett. B329(1994)399

[3] SMC, D.Adams et al., Phys.Lett. B357(1995)248

[4] SLAC E143, K.Abe et al., Phys.Rev.Lett. 74(1995)346

[5] SLAC E143, K.Abe et al., Phys.Rev.Lett. 75(1995)25

[6] R.L.Heimann, Nucl.Phys. B121(1977)445, J.Phys. G4(1978)173

[7] EMC, J.Ashman et al., Nucl.Phys. B328(1989)1

[8] SMC, B.Adeva et al., Nucl.Instr.Meth. A349(1994)334

[9] EMC, O.Allkofer et al., Nucl.Instr.Meth. 179(1981)445

[10] L.W.Whitlow et al., Phys.Lett. B250(1990)193

[11] L.L.Frankfurt et al., Phys.Lett. B230(1989)141

[12] A.D.Martin, W.J.Stirling and R.G.Roberts, Phys.Lett. 306B(1993)145

[13] EMC, M.Arneodo et al., Nucl.Phys. B321(1989)541

[14] SMC, B.Adeva et al., Phys.Lett. B320(1994)400

[15] NMC, P.Armaudruz et al., Phys.Rev.Lett. 66(1991)2712, M.Arneodo et al., Phys.Rev. D50(1994)1

[16] S.Kumano and J.T.Londergan, Phys.Rev. D44(1991)717

[17] P.Mulders, private communication

[18] M.Gluck, E.Reya and A.Vogt, Phys.Lett. B306(1993)391 
Table 1: Values of semi-inclusive asymmetries $A_{1 d}^{+}, A_{1 d}^{-}, A_{1 p}^{+}$and $A_{1 p}^{-}$and quark spin distributions $x \Delta u_{v}(x), x \Delta d_{v}(x)$ and $x \Delta \bar{q}(x)$. The spin distributions were obtained under the assumption $\Delta \bar{u}(x)=\Delta \bar{d}(x)$. The first errors are statistical and the second ones are systematic. The values of quark spins in the last three bins of $x$ correspond to the open circles in fig. 2.

\begin{tabular}{|c|c|c|c|c|c|}
\hline \multicolumn{6}{|c|}{$.8[.9]$} \\
\hline$\langle x\rangle$ & & $A_{1 d}^{+}(x)$ & & $A_{1 d}^{-}(x)$ & $A_{1 p}^{+}(x)$ \\
\hline 0.005 & \multicolumn{2}{|c|}{$0.033 \pm 0.070 \pm 0.006$} & \multicolumn{2}{|r|}{$0.086 \pm 0.077 \pm 0.009$} & $0.085 \pm 0.076 \pm 0.007$ \\
\hline 0.008 & \multicolumn{2}{|c|}{$-0.079 \pm 0.064 \pm 0.007$} & \multicolumn{2}{|c|}{$-0.134 \pm 0.071 \pm 0.010$} & $0.081 \pm 0.071 \pm 0.007$ \\
\hline 0.014 & \multicolumn{2}{|c|}{$-0.017 \pm 0.053 \pm 0.007$} & \multicolumn{2}{|c|}{$-0.203 \pm 0.058 \pm 0.014$} & $0.142 \pm 0.066 \pm 0.010$ \\
\hline 0.025 & \multicolumn{2}{|c|}{$-0.076 \pm 0.059 \pm 0.008$} & \multicolumn{2}{|c|}{$-0.071 \pm 0.068 \pm 0.008$} & $-0.026 \pm 0.089 \pm 0.006$ \\
\hline 0.035 & \multicolumn{2}{|c|}{$-0.015 \pm 0.083 \pm 0.008$} & \multicolumn{2}{|c|}{$-0.032 \pm 0.093 \pm 0.009$} & $0.009 \pm 0.105 \pm 0.006$ \\
\hline 0.049 & \multicolumn{2}{|c|}{$0.127 \pm 0.070 \pm 0.011$} & \multicolumn{2}{|c|}{$0.062 \pm 0.081 \pm 0.008$} & $0.204 \pm 0.090 \pm 0.014$ \\
\hline 0.077 & \multicolumn{2}{|c|}{$0.013 \pm 0.071 \pm 0.010$} & \multicolumn{2}{|r|}{$0.059 \pm 0.081 \pm 0.009$} & $0.175 \pm 0.087 \pm 0.013$ \\
\hline 0.12 & \multicolumn{2}{|c|}{$0.09 \pm 0.09 \pm 0.01$} & \multicolumn{2}{|r|}{$0.16 \pm 0.11 \pm 0.01$} & $0.54 \pm 0.11 \pm 0.03$ \\
\hline 0.17 & \multicolumn{2}{|c|}{$0.40 \pm 0.14 \pm 0.03$} & \multicolumn{2}{|r|}{$0.26 \pm 0.17 \pm 0.02$} & $0.34 \pm 0.16 \pm 0.02$ \\
\hline 0.24 & \multicolumn{2}{|c|}{$0.60 \pm 0.16 \pm 0.04$} & \multicolumn{2}{|r|}{$0.05 \pm 0.20 \pm 0.02$} & $0.37 \pm 0.18 \pm 0.02$ \\
\hline 0.34 & \multicolumn{2}{|c|}{$-0.29 \pm 0.30 \pm 0.03$} & \multicolumn{2}{|r|}{$-0.22 \pm 0.38 \pm 0.02$} & $0.29 \pm 0.31 \pm 0.02$ \\
\hline 0.48 & \multicolumn{2}{|c|}{$0.78 \pm 0.52 \pm 0.06$} & \multicolumn{2}{|r|}{$1.04 \pm 0.67 \pm 0.07$} & $0.93 \pm 0.48 \pm 0.06$ \\
\hline \multirow{13}{*}{$.8[.9]$} & \multicolumn{3}{|c|}{$x \Delta u_{v}(x)$} & $x \Delta d_{v}(x)$ & $x \Delta \bar{q}(x)$ \\
\hline & 0.005 & $0.05 \pm 0.15 \pm 0$ & & $-0.14 \pm 0.17 \pm 0.02$ & $0.027 \pm 0.075 \pm 0.002$ \\
\hline & 0.008 & $0.14 \pm 0.13 \pm 0$ & & $-0.13 \pm 0.14 \pm 0.02$ & $-0.018 \pm 0.062 \pm 0.004$ \\
\hline & 0.014 & $0.24 \pm 0.10 \pm 0$ & & $0.07 \pm 0.11 \pm 0.02$ & $-0.085 \pm 0.047 \pm 0.012$ \\
\hline & 0.025 & $-0.01 \pm 0.11 \pm 0$ & & $-0.16 \pm 0.13 \pm 0.02$ & $0.040 \pm 0.053 \pm 0.005$ \\
\hline & 0.035 & $0.04 \pm 0.14 \pm 0$ & & $-0.13 \pm 0.16 \pm 0.02$ & $0.027 \pm 0.067 \pm 0.004$ \\
\hline & 0.049 & $0.22 \pm 0.12 \pm 0$ & & $0.08 \pm 0.13 \pm 0.02$ & $-0.045 \pm 0.056 \pm 0.007$ \\
\hline & 0.077 & $0.10 \pm 0.11 \pm 0$ & & $-0.24 \pm 0.13 \pm 0.02$ & $0.047 \pm 0.054 \pm 0.005$ \\
\hline & 0.12 & $0.25 \pm 0.14 \pm 0$ & & $-0.21 \pm 0.17 \pm 0.02$ & $0.022 \pm 0.067 \pm 0.004$ \\
\hline & 0.17 & $0.24 \pm 0.19 \pm 0$ & & $0.15 \pm 0.22 \pm 0.04$ & $-0.020 \pm 0.090 \pm 0.007$ \\
\hline & 0.24 & $0.21 \pm 0.07 \pm 0$ & & $-0.03 \pm 0.10 \pm 0.08$ & \\
\hline & 0.34 & $0.34 \pm 0.09 \pm 0$ & & $-0.25 \pm 0.12 \pm 0.03$ & \\
\hline & 0.48 & $0.19 \pm 0.06 \pm 0$ & & $-0.12 \pm 0.09 \pm 0.01$ & \\
\hline
\end{tabular}


Table 2: Values of the correlation coefficients between semi-inclusive asymmetries, $\operatorname{corr}\left(A_{1}^{+}, A_{1}^{-}\right)$, and between inclusive and semi-inclusive asymmetries, $\operatorname{corr}\left(A_{1}, A_{1}^{+}\right)$and $\operatorname{corr}\left(A_{1}, A_{1}^{-}\right)$, for deuteron and proton.

\begin{tabular}{c|c|c|c|c|c|c}
\multicolumn{7}{c}{$.8[.9]$} \\
\hline \hline$\langle x\rangle$ & $\operatorname{corr}\left(A_{1 d}^{+}, A_{1 d}^{-}\right)$ & $\operatorname{corr}\left(A_{1 d}, A_{1 d}^{+}\right)$ & $\operatorname{corr}\left(A_{1 d}, A_{1 d}^{-}\right)$ & $\operatorname{corr}\left(A_{1 p}^{+}, A_{1 p}^{-}\right)$ & $\operatorname{corr}\left(A_{1 p}, A_{1 p}^{+}\right)$ & $\operatorname{corr}\left(A_{1 p}, A_{1 p}^{-}\right)$ \\
\hline 0.005 & 0.13 & 0.32 & 0.29 & 0.13 & 0.33 & 0.30 \\
0.008 & 0.12 & 0.32 & 0.29 & 0.13 & 0.37 & 0.33 \\
0.014 & 0.13 & 0.34 & 0.30 & 0.14 & 0.40 & 0.37 \\
0.025 & 0.13 & 0.36 & 0.32 & 0.15 & 0.44 & 0.39 \\
0.035 & 0.13 & 0.37 & 0.33 & 0.15 & 0.46 & 0.41 \\
0.049 & 0.12 & 0.39 & 0.34 & 0.16 & 0.48 & 0.41 \\
0.077 & 0.12 & 0.40 & 0.34 & 0.18 & 0.50 & 0.41 \\
0.12 & 0.12 & 0.38 & 0.31 & 0.16 & 0.47 & 0.37 \\
0.17 & 0.11 & 0.36 & 0.28 & 0.14 & 0.42 & 0.32 \\
0.24 & 0.11 & 0.30 & 0.24 & 0.12 & 0.35 & 0.26 \\
0.34 & 0.11 & 0.25 & 0.19 & 0.12 & 0.28 & 0.21 \\
0.48 & 0.10 & 0.19 & 0.15 & 0.13 & 0.22 & 0.16 \\
\hline \hline
\end{tabular}

Table 3: Values of integrals over $x$ of the valence and sea quark spin distributions. The spin distributions were obtained under the assumption $\Delta \bar{u}(x)=\Delta \bar{d}(x)$. Contributions from the large- $x$ region are negligible. In the small-x region the error is systematic. In the other regions the first error is statistical and the second one is systematic.

\begin{tabular}{|c|c|c|c|c|}
\hline \multirow{5}{*}{$.8[.9]$} & $x$ & $0 \div 0.003$ & $0.003 \div 0.7$ & $0 \div 1$ \\
\hline & $\Delta u_{v}$ & $0.11 \pm 0.11$ & $0.90 \pm 0.19 \pm 0.09$ & $1.01 \pm 0.19 \pm 0.14$ \\
\hline & $\Delta d_{v}$ & $-0.06 \pm 0.06$ & $-0.50 \pm 0.22 \pm 0.09$ & $-0.57 \pm 0.22 \pm 0.11$ \\
\hline & $x$ & $0 \div 0.003$ & $0.003 \div 0.2$ & $0 \div 1$ \\
\hline & $\Delta \bar{q}$ & $0.00 \pm 0.02$ & $-0.02 \pm 0.09 \pm 0.02$ & $-0.02 \pm 0.09 \pm 0.03$ \\
\hline
\end{tabular}




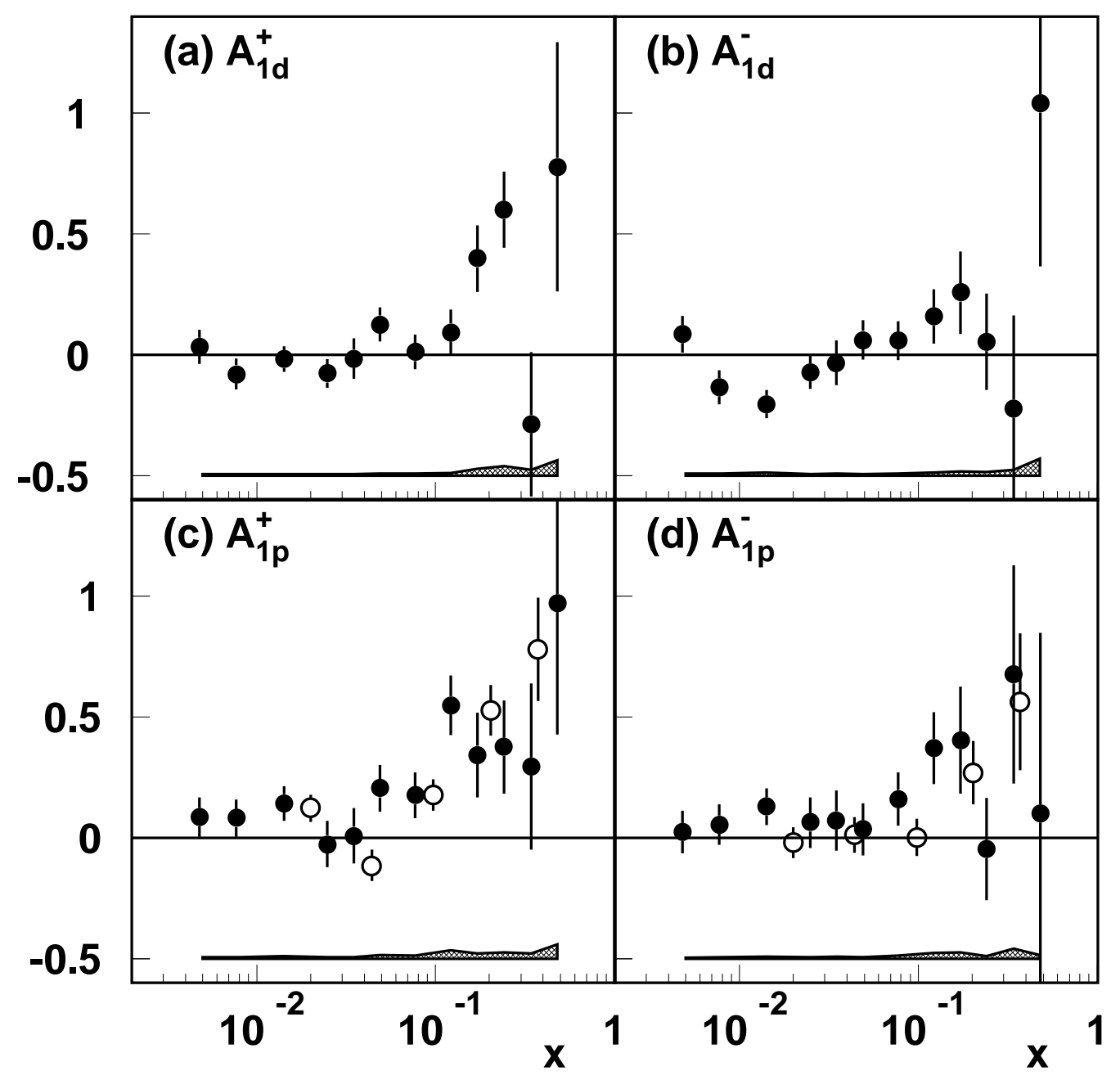

Figure 1: Semi-inclusive asymmetries of spin-dependent cross sections for muoproduction of (a) positive hadrons on deuteron, (b) negative hadrons on deuteron, (c) positive hadrons on proton and (d) negative hadrons on proton. The error bars are statistical and the shaded areas represent the systematic uncertainty. The open circles represent the asymmetries measured by EMC [7]. 


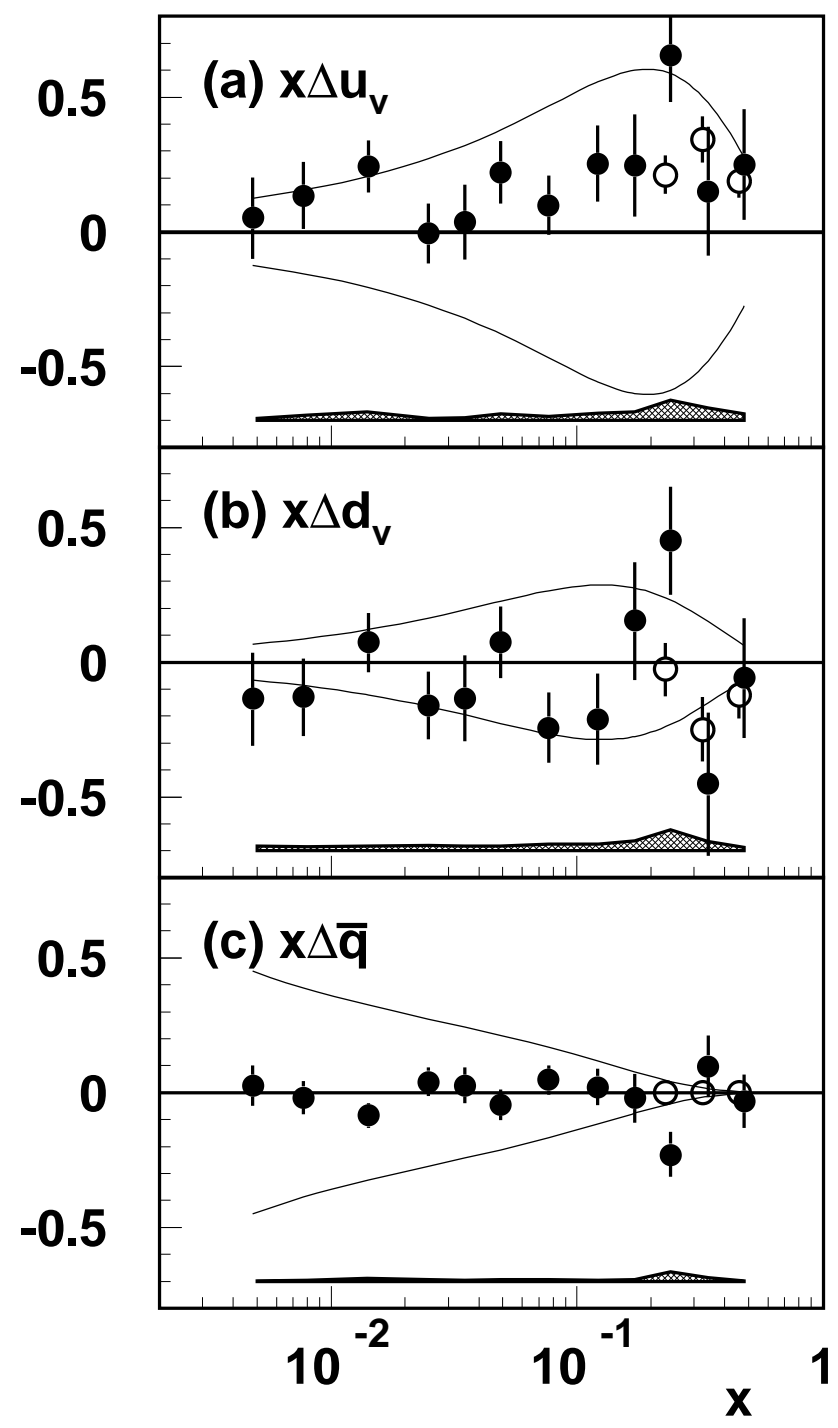

Figure 2: Quark spin distribution functions (a) $x \Delta u_{v}(x)$, (b) $x \Delta d_{v}(x)$, (c) $x \Delta \bar{q}(x)$ obtained under the assumption $\Delta \bar{u}(x)=\Delta \bar{d}(x)$. The open circles are obtained when the sea polarisation is set to zero while the closed circles are obtained without this assumption. The error bars are statistical and the shaded areas represent the systematic uncertainty. The curves correspond to the upper and the lower limits $\pm q(x)$ from the unpolarised quark distributions [12] evaluated at $Q^{2}=10 \mathrm{GeV}^{2}$. 


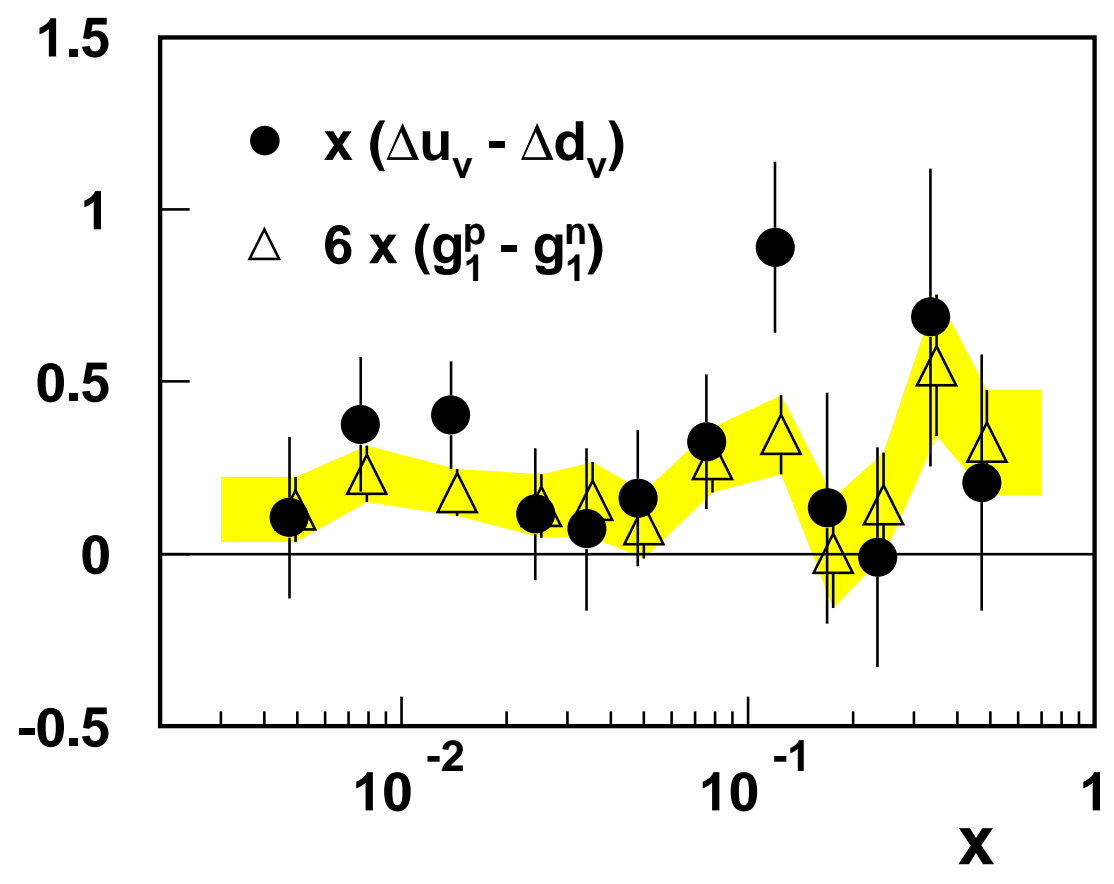

Figure 3: The difference between the spin-dependent structure functions of the proton and the neutron, $6 x\left[g_{1}^{p}(x)-g_{1}^{n}(x)\right]$, (open triangles and shaded belt), and the difference between up and down valence quark spin distribution functions $x\left[\Delta u_{v}(x)-\Delta d_{v}(x)\right]$, as determined from SMC semi-inclusive asymmetries only under the assumption $\Delta \bar{u}(x)=\Delta \bar{d}(x)$ (closed circles). Errors are statistical only. 

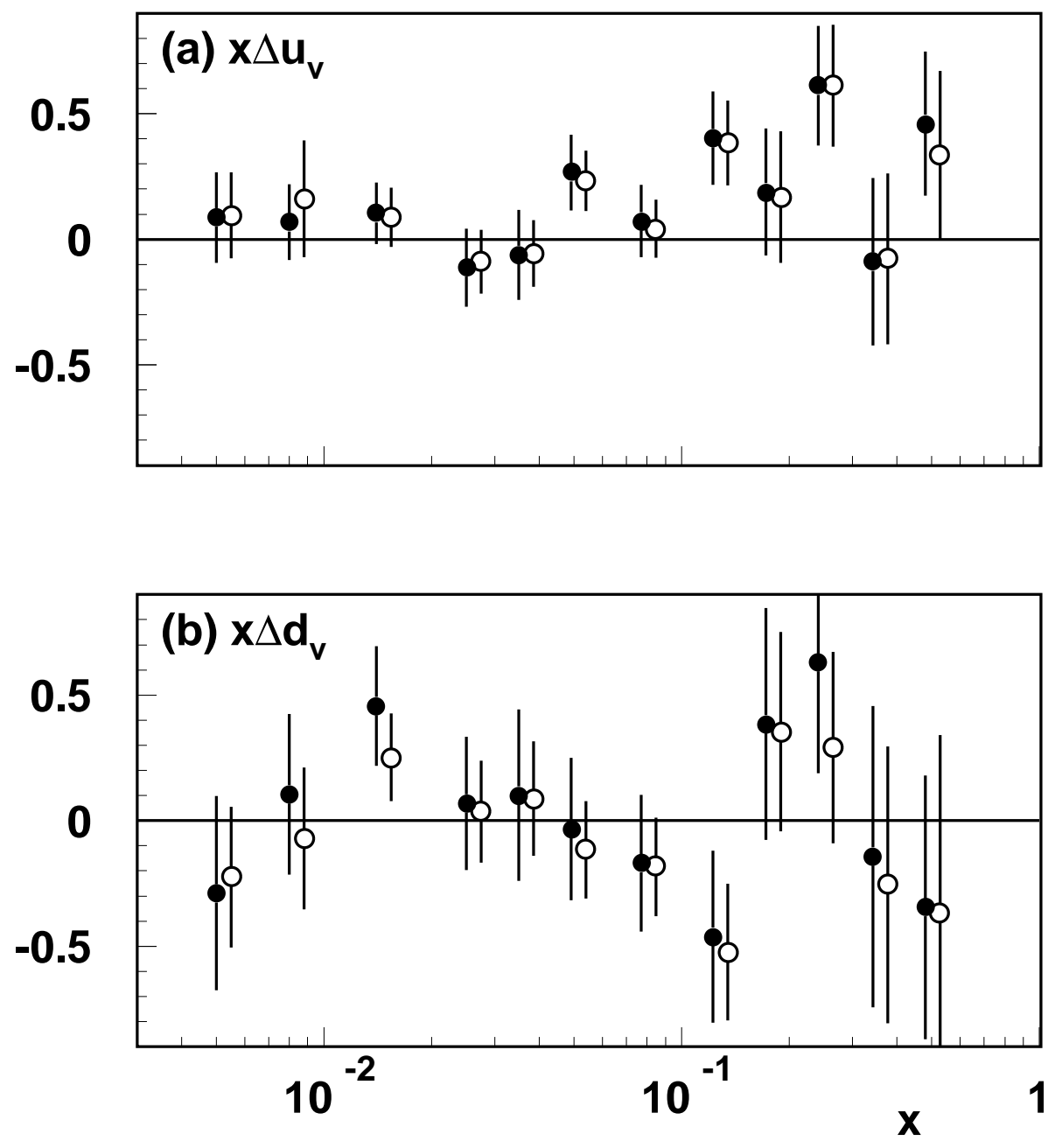

Figure 4: Quark spin distribution functions (a) $x \Delta u_{v}(x)$ and (b) $x \Delta d_{v}(x)$ obtained from the asymmetries $A^{+-}$(eq. (6), open circles) and the asymmetries $A^{+}$and $A^{-}$(eq. (2), closed circles) without using inclusive asymmetries and without the constraint $\Delta \bar{u}=\Delta \bar{d}$. Errors are statistical. 